\title{
Effect of Nozzle Geometry and Distance on Cooling Performance of Impinging Jets
}

\author{
Hirokazu KOBAYASHI, ${ }^{*}$ Kazuhisa KABEYA, Yukio TAKASHIMA, Hideyuki TAKAHASHI and Gentaro TAKEDA \\ Steel Research Laboratory, JFE Steel Corporation, 1, Kawasaki-dori, Mizushima, Kurashiki-shi, Okayama, $712-8511$ Japan. \\ (Received on March 14, 2018; accepted on April 19, 2018; originally published in Tetsu-to-Hagané, Vol. \\ 103, 2017, No. 8, pp. 458-467)
}

\begin{abstract}
Gas jet cooling is widely used because the device is simple, it is possible to prevent oxidation and a uniform cooling ability can be obtained with thin steel sheets.

Because the gas jet cooling ability is affected by the gas properties and nozzle geometry, quantitative evaluation of the influences of these factors is very important.

Several non-dimensional empirical equations have been proposed for prediction of the gas jet cooling ability of geometrically arranged nozzles. In this research, the accuracy of Martin's non-dimensional empirical equations was investigated by gas jet cooling experiments and fluid analyses with different nozzle geometries and distances from the cooled surface.

Two nozzle geometries were examined, namely, a slit-like (linear) arrangement of round nozzles and a staggered arrangement of round nozzles.

The difference between the experimental results and the results of predictions by Martin's equations increased as the distance between the nozzle tips and the cooled surface decreased. It was suggested that the characteristic difference of the jet flow between the flow development region (potential core region) and the fully developed flow region influenced the cooling ability.

The trend of divergence between the experimental and predicted cooling ability was clearly presented in this research. The results of this study make it possible to improve the accuracy of predictions when the distance between the nozzle tips and the cooled surface is set within the potential core region of the jet.
\end{abstract}

KEY WORDS: gas jet cooling; nozzle geometry; heat transfer coefficient; contracted flow; flow development region; potential core.

\section{Introduction}

Gas jet cooling, which promotes heat transfer by utilizing forced convection by gas injection, is widely used to cool steel strips in the annealing process in steel manufacturing. The advantages of gas jet cooling are that oxidation can be prevented by recycling the reducing atmosphere gas in the annealing furnace, and excellent cooling uniformity can be obtained because the temperature of the cooling surface does not affect the cooling ability, unlike the boiling phenomenon that occurs with water cooling.

The cooling ability of the gas jet affects various results such as improvement of productivity, improvement of properties such as the strength and workability of the steel sheet, reduction of the added alloy cost, the compactness of the equipment and energy saving. Therefore, prediction of the cooling ability of the gas jet is very important in nozzle design. However, because many factors such as the nozzle shape, arrangement, distance, header pressure and radiation influence the cooling ability, accurate prediction is difficult.

\footnotetext{
* Corresponding author: E-mail: hirok-kobayashi@jfe-steel.co.jp DOI: http://dx.doi.org/10.2355/isijinternational.ISIJINT-2018-133
}

Many studies in the literature have presented detailed reports on the factors that influence the cooling ability of a single nozzle. For example, Choo et al. ${ }^{1)}$ investigated the effect of pressure loss due to nozzle proximity under constant pressure, and Prabhu et al. ${ }^{2)}$ studied the effect of the shape of the nozzle tip on cooling performance.

On the other hand, many studies have also examined the cooling ability of multi-hole nozzles, with which it is necessary to consider the geometric arrangement of the nozzles. For example, Martin's research ${ }^{3)}$ and Omori's experiment, ${ }^{4)}$ which formulated prediction equations for the heat transfer coefficient considering experimental verification and a shape function, were referenced and verified in many subsequent research reports. ${ }^{5,6)}$

In order to improve the cooling ability in gas jet cooling, positioning the nozzle close to the substrate to be cooled is often considered. Nozzle proximity has the advantage of reducing energy costs, as it is not necessary to increase the operation load of the blower to increase the gas flow rate in order to increase the cooling ability. However, few studies have attempted an in-depth verification of the cooling performance of multi-hole nozzles in the flow development region where the potential core ${ }^{5,6)}$ exists. For example, 
Atallaa et $a l .{ }^{7)}$ confirmed that the nozzle distance has little effect on heat transfer in the region of $2 \leqq H / D \leqq 5$, where the distance between the substrate to be cooled and the nozzle is $\mathrm{H}$ and the nozzle diameter is D. In addition, Narayanan et $a{ }^{8}{ }^{8}$ mentioned that turbulence outside the impinging jet caused unsteadiness of the thermal boundary layer and promoted heat transfer in an experiment concerning the proximity of a slit nozzle.

As described above, there are numerous examples of verification of the gas flow and cooling ability of multi-hole nozzles and many cooling capacity prediction equations have been formulated, but the conditions and nozzle arrangements are often limited. On the other hand, Martin et $a l^{3)}$ formulated a systematic prediction formula that considered various nozzle shapes (slits and round holes) and geometric arrangements (staggered arrangement and grid arrangement), and this formula is very beneficial in practical use.

Martin proposed a dimensionless characteristic equation that expressed the relationship between the Nusselt number, Reynolds number, Prandtl number and nozzle shape function (consisting of the nozzle distance, diameter, arrangement, etc.) based on cooling experiments. This dimensionless characteristic equation for multi-hole nozzles is extremely convenient because it can easily predict the gas jet cooling ability without a complicated gas flow analysis. However, details such as error, accuracy and the tendency of divergence in the range of application had not been sufficiently clarified.

Therefore, in order to improve the accuracy of the simple prediction formula of the gas jet cooling ability of multi-hole nozzles, in this study, we conducted cooling experiments and numerical analyses with various nozzle distances, arrangements, nozzle heights and nozzle diameters, and clarified the effect of each factor on the cooling ability. Furthermore, we conducted an accuracy verification of the Martin prediction formula and considered guidelines to improve the prediction accuracy of the gas jet cooling ability.

\section{Experimental Set-up and Procedure}

Figure 1 shows the outline of the gas jet cooling system used in this experiment. As the substrate, SUS 304 (size: L $250 \times \mathrm{W} 100 \times \mathrm{t} 10[\mathrm{~mm}])$ was used in order to exclude the effect of transformation heat generation.

As the experimental procedure, the SUS 304 substrate is heated to $600^{\circ} \mathrm{C}$ in a furnace and set on the stand above the nozzle. The internal air pressure is checked with a pressure gauge attached to the nozzle header, and is adjusted by using a valve attached to the gas pipe. When the temperature of the substrate reaches $550^{\circ} \mathrm{C}$, the masking plate between the

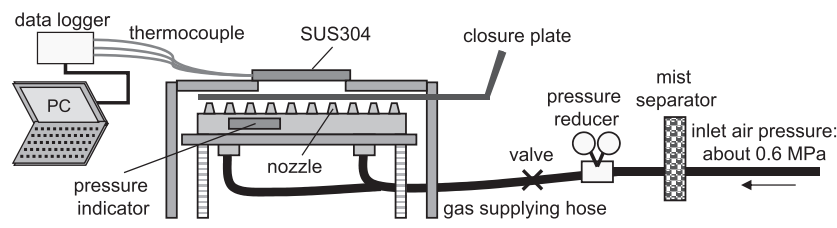

Fig. 1. Schematic illustration of experimental setup.
SUS and the nozzle is opened and cooling is started.

The temperature of the SUS was measured with K type thermocouples $(\phi 0.5 \mathrm{~mm})$. Figure 2 shows the relationship between the positions of the thermocouples in the SUS and the nozzle positions. Two nozzle conditions were examined, the first being a slit-like nozzle with circular holes linearly arranged at short pitches, and second a nozzle with a staggered arrangement in which the distance between each circular nozzle was the same. The thermocouples were set from $\phi 0.5 \mathrm{~mm}$ holes created by electric discharge machining on the side surface of the SUS, and the depth from the cooling surface was $2 \mathrm{~mm}$. The thermocouples were set at three points, namely, the stagnation point of the nozzle and at $1 / 2$ and $1 / 4$ of the distance between the nozzles. The average temperature of these three points was evaluated as the in-plane average temperature of the $2 \mathrm{~mm}$ depth from the cooling surface. The average heat transfer coefficients of the cooling surface from $500^{\circ} \mathrm{C}$ to $300^{\circ} \mathrm{C}$ were calculated from the average heat history.

In calculating the heat transfer coefficients, the radiation of the SUS and heat transfer on the back side of the cooling surface were considered. The emissivity was assumed as 0.8 , and the heat transfer coefficient of the back surface was assumed as $11.63 \mathrm{~W} / \mathrm{m}^{2} \mathrm{~K}$. The emissivity of a glossy SUS surface is generally lower that this value. However, in this experiment, the surface was oxidized and blackish because the SUS was repeatedly heated to $600^{\circ} \mathrm{C}$. Therefore, emissivity was set to 0.8 , which is a value close to the emissivity of the oxidized surface. ${ }^{9)}$ In natural convection with a steel surface temperature in the range from $300^{\circ} \mathrm{C}$ to $500^{\circ} \mathrm{C}$, the maximum value of the heat transfer coefficient is about $11.63 \mathrm{~W} / \mathrm{m}^{2} \mathrm{~K}$. ${ }^{9}$ In addition, because the experiment was conducted in a building without air-conditioning during the Japanese summer, the atmosphere and jet air temperatures were set at $30^{\circ} \mathrm{C}$.

Table 1 shows the experimental conditions. Two nozzle header pressures conditions were used, $3 \mathrm{kPa}$ and $5 \mathrm{kPa}$, which are a range generally used for gas jet cooling in CAL (Continuous Annealing Line) and CGL (Continuous Galvanizing Line) facilities. Two types of nozzles were investigated, as shown in Fig. 3, a slit-like nozzle in which round holes are linearly arranged at a short pitch and a staggered arrangement nozzle in which the distances between the nozzles are the same. In the slit-like nozzle, the nozzle holes are arranged at narrow spans in the width direction (lateral direction of Fig. 3), which has the advantage that the temperature distribution in the width direction can be made
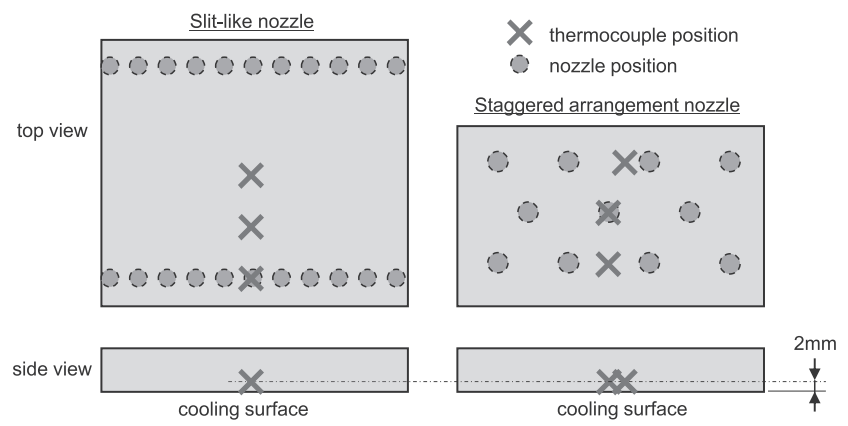

Fig. 2. Thermocouple arrangement and nozzle position. 
uniform, like that with true slit nozzle. On the other hand, with the staggered arrangement nozzle, the temperature difference increases due to the long spans between the nozzles in the width direction. However, because the nozzles are arranged at the same spans, cooling efficiency is high because the area which one nozzle cools is optimized, that is, the distance cooled by the lateral flow of the impinging jet is almost same in all directions.

The opening area ratios of the nozzles are 0.012 in the slit-like nozzle and 0.018 in the staggered arrangement nozzle. The aperture ratio of the nozzle was defined as the ratio of the nozzle opening area and the area to be cooled for one nozzle. In the slit nozzle, a 25A carbon steel pipe with a thickness of $3.2 \mathrm{~mm}$ was used for the nozzle header, and a round holes with a size of $\phi 3.2 \mathrm{~mm}$ were made at a span of $5.6 \mathrm{~mm}$. In the staggered arrangement nozzle, a long cone nozzle is attached to a box-shaped nozzle header. The nozzle height was $10 \mathrm{~mm}$, the opening diameter of the nozzle base was $\phi 6 \mathrm{~mm}$, and the opening diameter of the nozzle tip was $\phi 4 \mathrm{~mm}$. The nozzle had a tapered shape. Because this tapered nozzle design reduces pressure loss compared with a straight nozzle, it is possible to decrease the blower load and increase the gas flow rate at the same header pressure. In addition, velocity attenuation is reduced because the straightness of the jet is improved compared with a simple round nozzle without height. Since these nozzles have height, it is also possible to increase the space between the nozzle header and the object to be cooled, which improves cooling efficiency by promoting the discharge of the high temperature gas. As a disadvantage, because the tapered nozzle has a complicated shape, the nozzle production cost is high.

On the other hand, since the slit-like nozzle consists of simple round holes, it is easier to produce than an ordinary slit nozzle, and because the opening does not extend across the full width of the nozzle, the strength of the nozzle header is high and deformation resistance is good. However, because there is no prediction equation for the heat transfer

Table 1. Experimental conditions.

\begin{tabular}{ccc}
\hline $\begin{array}{c}\text { Nozzle } \\
\text { arrangement }\end{array}$ & Slit-like & $\begin{array}{c}\text { Staggered } \\
\text { arrangement }\end{array}$ \\
\hline Pressure & $3,5 \mathrm{kPa}$ & $3,5 \mathrm{kPa}$ \\
Nozzle distance & $10-30 \mathrm{~mm}$ & $10-30 \mathrm{~mm}$ \\
Nozzle diameter & $3.2 \mathrm{~mm}$ & $4.0 \mathrm{~mm}$ \\
Nozzle shape & Circle & Circle \\
Nozzle pitch & $\begin{array}{c}\text { Width direction: } 5.6 \mathrm{~mm} \\
\text { Length direction: } 120 \mathrm{~mm}\end{array}$ & $\begin{array}{c}\text { Width direction: } 28 \mathrm{~mm} \\
\text { Length direction: } 24 \mathrm{~mm}\end{array}$ \\
\hline
\end{tabular}
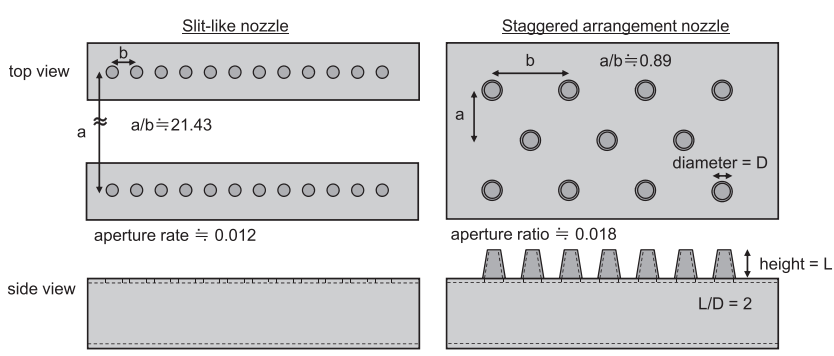

Fig. 3. Nozzle arrangement and shape. coefficient of slit-like nozzles with multiple holes, new consideration is necessary.

Therefore, we investigated cooling performance by experiments with two types of nozzles having these characteristics, and attempted to improve the accuracy of cooling ability prediction by understanding the effect of each factor and comparing the results with Martin's prediction formula.

\section{Effect of Nozzle Geometry}

\subsection{Results and Discussion}

Figure 4 shows the relationship of the heat transfer coefficients in the experiment and the results of Martin's prediction formula. $H$ is the distance between the nozzle tip and the object to be cooled. Because there is no prediction formula for the slit-like nozzle, a slit nozzle having the equivalent nozzle opening area was assumed. Figure 4 shows the value calculated by the Martin prediction formula for a slit gap of $1.436 \mathrm{~mm}$.

Next, Martin's dimensionless characteristic equations are shown in (1) and (2). In the staggered arrangement nozzles, formula (1) is applied in the range of $2000 \leqq R e \leqq 100000$, and in the slit nozzles, formula (2) is applied in the range of $1500 \leqq \mathrm{Re} \leqq 40000$. The average heat transfer coefficient $H_{m}$ can be calculated by formulas (3) and (4).

$$
\begin{aligned}
& \mathrm{Nu}_{m}=K \cdot \sqrt{f} \frac{1-2.2 \sqrt{f}}{1+0.2\left(\frac{H}{D}-6\right) \sqrt{f}} \cdot \operatorname{Re}^{\frac{2}{3}} \cdot \operatorname{Pr}^{0.42} \\
& \mathrm{Nu}_{m}=\frac{2}{3} \cdot f_{0}^{\frac{3}{4}}\left(\frac{2 \mathrm{Re}}{f / f_{0}+f_{0} / f}\right)^{\frac{2}{3}} \cdot \operatorname{Pr}^{0.42} \\
& H_{m}=\frac{\mathrm{Nu}_{m} \cdot \lambda}{D} \text {. }
\end{aligned}
$$

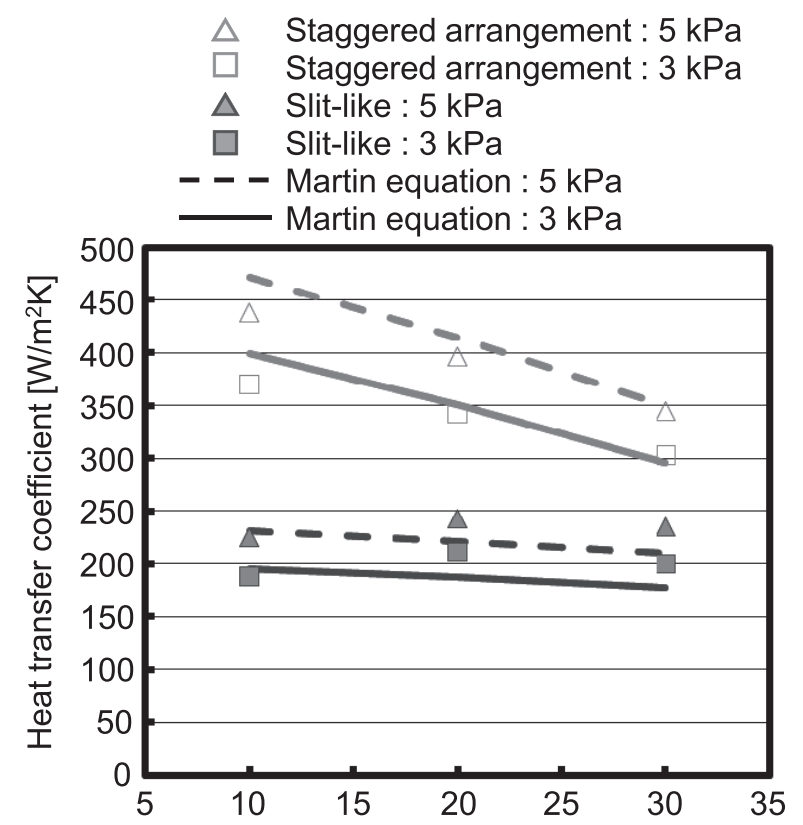

$\mathrm{H}$ : distance between nozzle exit and SUS surface [mm]

Fig. 4. Comparison of heat transfer coefficient in experiment and results of Martin equation. 


$$
H_{m}=\frac{\mathrm{Nu}_{m} \cdot \lambda}{B}
$$

$D$ is the nozzle diameter, $\lambda$ is the thermal conductivity of the gas, Re is the Reynolds number, Pr is the Prandtl number, $\mathrm{Nu}_{m}$ is the average Nusselt number, $B$ is the nozzle slit width and $H_{m}$ is the average heat transfer coefficient.

$f$ and $K$ are the shape functions of the nozzle, and are expressed by formulas (5) and (8) for the staggered arrangement nozzle and by formulas (6) and (7) for the slit nozzle.

$$
\begin{array}{r}
f=\frac{\pi}{2 \sqrt{3}}\left(\frac{D}{L}\right)^{2} \ldots \ldots \ldots \ldots . . . \\
f=\frac{B}{L} \ldots \ldots \ldots \ldots \ldots \ldots \ldots \\
f_{0}=\left[60+4\left(\frac{H}{2 B}-2\right)^{2}\right]^{-0.5} \ldots \\
K=\left[1+\left(\frac{H / D}{0.6 / \sqrt{f}}\right)^{6}\right]^{-0.05} \ldots \ldots
\end{array}
$$

where, $L$ is the distance of nozzle span and $H$ is the distance between the nozzle tip and the object to be cooled. Table 2 shows the values of the shape functions $f, f_{0}$ and $K$ at each nozzle distance.

The definition of the Reynolds number is shown below.

$$
\operatorname{Re}=\frac{w S}{v}
$$

Here, $w$ is the gas velocity at the nozzle exit and $v$ is the viscosity coefficient. $S$ is $D$ in the staggered arrangement nozzles, and $S$ is $2 B$ in the slit-like nozzles.

In the experimental results in Fig. 4, the cooling ability of the staggered nozzle is higher than that of the slit-like nozzle, and the cooling ability increases with higher header pressure. These are appropriate results, as shown by the relationship of the nozzle aperture ratio and the air volume rate. On the other hand, in case of nozzle proximity, the cooling ability improved with the staggered nozzle and decreased with the slit-like nozzle.

In Fig. 4, the calculation results of the Martin equation of the staggered nozzle plot the value at the nozzle diameter $\phi 4 \mathrm{~mm}$. Although these values are almost the same as the experimental values, the improvement of the cooling ability by nozzle proximity was overestimated by about $10 \%$ at

Table 2. Numerical values of nozzle geometry function.

\begin{tabular}{ccccc}
\hline & \multicolumn{2}{c}{$\begin{array}{c}\text { Slit-like } \\
\text { B: } 1.436[\mathrm{~mm}]\end{array}$} & \multicolumn{2}{c}{$\begin{array}{c}\text { Staggered arrangement } \\
\text { D: } 4[\mathrm{~mm}]\end{array}$} \\
\hline $\begin{array}{c}\text { Nozzle } \\
\text { distance }\end{array}$ & $f$ & $f_{0}$ & $f$ & $K$ \\
$10 \mathrm{~mm}$ & 0.01197 & 0.12058 & 0.01851 & 0.99837 \\
$20 \mathrm{~mm}$ & 0.01197 & 0.07942 & 0.01851 & 0.94465 \\
$30 \mathrm{~mm}$ & 0.01197 & 0.05381 & 0.01851 & 0.85103 \\
\hline
\end{tabular}

the nozzle distance of $10 \mathrm{~mm}$. Although a contraction flow usually occurs in a jet flow, the Martin prediction formula is defined by the actual nozzle diameter, and the contraction flow is not considered. In this experiment, in the case of the tapered nozzles, that is, under a condition with almost no effect of the contraction flow, the heat transfer coefficients were almost the same as those given by the Martin prediction formula.

With the slit-like nozzle, the Martin prediction formula showed a lower heat transfer coefficient than the experimental value when the nozzle distance was long. In the case of nozzle proximity, the Martin prediction formula tended to overestimate the improvement in cooling ability. However, in the experiment, the heat transfer coefficient was smallest at the nozzle distance of $10 \mathrm{~mm}$. As described above, with the staggered arrangement nozzle and the slit-like nozzle, when the results of the experiment and the Martin prediction formula were compared, it became clear that there are differences in the value and tendency of the heat transfer coefficient depending on the nozzle distance. With both types of nozzles, the Martin prediction formula tended to overestimate the improvement of the cooling ability by nozzle proximity. Regarding this tendency, Attalla et al. ${ }^{7}$ reported that the ratio of $\mathrm{H}$ and $\mathrm{D}$ had little effect on heat transfer in the range of $2 \leqq \mathrm{H} / \mathrm{D} \leqq 5$ in an experiment with a grid arrangement nozzle. Geers et al. ${ }^{10)}$ also reported that there was virtually no change in heat transfer in the range of $\mathrm{H} / \mathrm{D} \leqq 4$ in an experiment with a staggered arrangement nozzle. Therefore, the results of this experiment are considered appropriate.

With the slit-like nozzle, the reason for the increase in the heat transfer coefficient in the experiment in comparison with the Martin prediction equation (prediction equation for slit nozzle) is considered to be the effect of the contraction flow. Omori et al. ${ }^{4)}$ showed that the contraction ratios of a multi-hole nozzle and a multiple slit nozzle depend only on the nozzle span, and proposed the following equations. Multi-hole nozzle

$$
d / D=12 x_{n}^{2}-3.19 x_{n}+1 \quad x_{n} \leq 0.133
$$

Multiple slit nozzle

$$
b / B=11.1 x_{n}^{2}-2.67 x_{n}+0.89 x_{n} \leq 0.12
$$

where, $\mathrm{d}$ and $\mathrm{b}$ are the true jet diameter and width, $\mathrm{D}$ and $\mathrm{B}$ are the nozzle diameter and the slit width and $x_{n}$ is the nozzle span (unit: meter). Under the experimental conditions used in the present study, the contraction flow ratio of the slit-like nozzle (multi-hole nozzle) is about 0.98 , and the contraction flow ratio of the slit nozzle is 0.73 . Therefore, the ratio of both is about 1.34 . When the slit width in the Martin prediction formula is $1.92 \mathrm{~mm}(1.436[\mathrm{~mm}] \times 1.34)$, the result is close to the experimental results for a nozzle distance of 20-30 mm. Thus, in predicting the cooling ability of a slit-like nozzle, this study clarified the fact that prediction accuracy can be improved by considering the contraction ratio in the Martin prediction equation (slit type nozzle). However, when the nozzle distance is set to $10 \mathrm{~mm}$, the decrease in the cooling ability cannot be explained by the contraction ratio.

Figure 5 shows the results of temperature measurements by the thermocouples. In order to compare these results, 

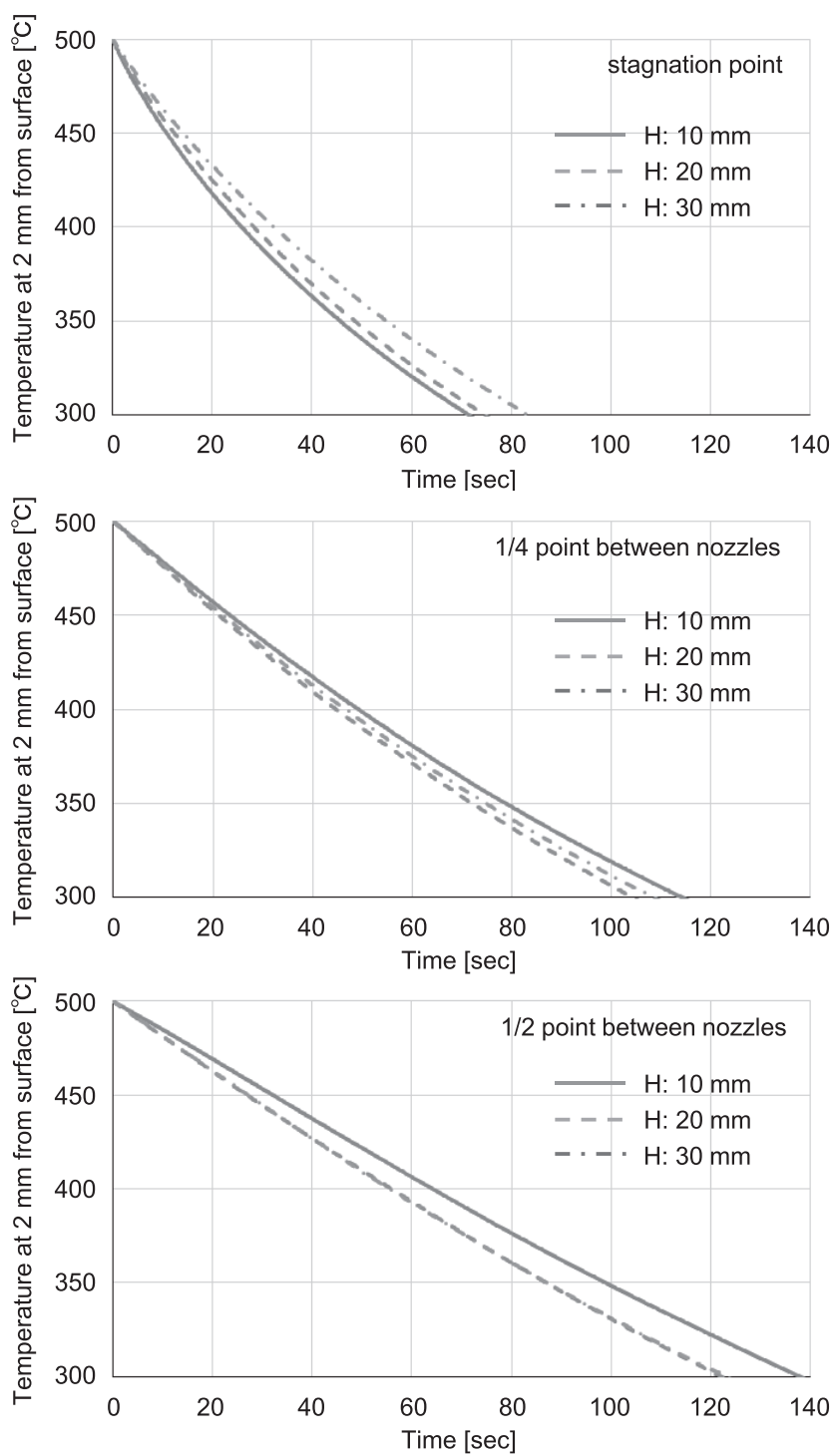

Fig. 5. Temperature history in experiment (slit-like nozzle: 3 $\mathrm{kPa})$.

$500^{\circ} \mathrm{C}$ at each measurement position is set to $0 \mathrm{sec}$. At the stagnation point, the cooling rate is the largest when the nozzle distance $\mathrm{H}$ is small $(10 \mathrm{~mm})$. However, at the $1 / 4$ point and $1 / 2$ point between the nozzles, the cooling rate is the largest when the nozzle distance is long $(30 \mathrm{~mm})$. That is, with the slit-like nozzle, it can be understood that the decrease in cooling ability due to nozzle proximity occurred as a result of a decrease in the cooling ability between the nozzles. The lateral flow along the surface after the jet impinges also contributes to cooling. Omori et al. ${ }^{4)}$ also suggest that considerable heat transfer occurs between the nozzles in multi-hole nozzles. Therefore, when the cooling ability between the nozzles decreases, the average heat transfer decreases drastically.

Next, in the case of the staggered arrangement nozzle, the temperature measurement results of the thermocouples are shown in Fig. 6. In order to compare the results, $500^{\circ} \mathrm{C}$ at each measurement position is also set to $0 \mathrm{sec}$ in this case. Regardless of whether the measurement position is at the stagnation point or between the nozzles, the heat history is almost the same. In addition, the cooling ability also increases as the nozzle distance becomes closer, and
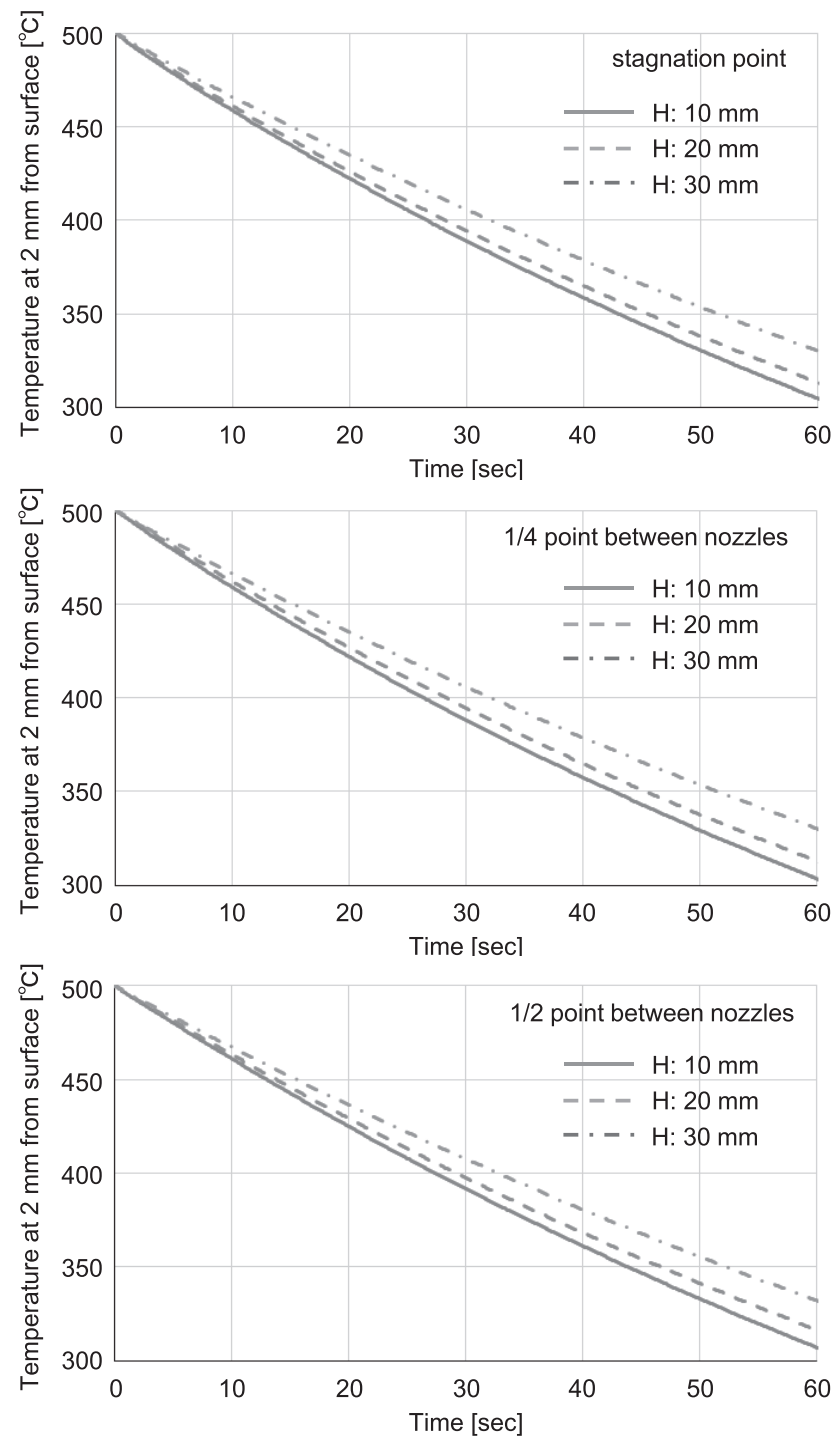

Fig. 6. Temperature history in experiment (staggered arrangement nozzle: $3 \mathrm{kPa}$ ).

a remarkable decrease in the cooling rate between nozzles due to nozzle proximity, like that with the slit-like nozzle, did not occur.

In order to evaluate the cooling by the lateral flow of gas on the surface being cooled, the results of the experiment were organized by dimensionless numbers. The Reynolds number $\mathrm{Re}$ and Nusselt number $\mathrm{Nu}$ are generally used to evaluate heat transfer by forced convection. In this research, as in Omori et al., ${ }^{4}$ the representative velocity and length are expressed as the collision velocity of the jet and the nozzle span, respectively.

$$
\begin{aligned}
\mathrm{R} e_{a} & =\frac{u_{a} \cdot x_{n}}{v} \\
\mathrm{Nu}_{a v} & =\frac{\alpha_{a v} \cdot x_{n}}{\lambda}
\end{aligned}
$$

where, $u_{a}$ is the collision velocity of the jet, $x_{n}$ is the nozzle span, $v$ is the kinematic viscosity coefficient and $\alpha_{a v}$ is the average heat transfer coefficient. The collision velocity of the jet was measured at each nozzle distance by a hot wire gas flow meter. The measured data organized by the mov- 
ing average of 20 points are shown in Figs. 7 and 8. The collision velocity was defined as the average velocity in the width direction in the slit-like nozzle, and as the average velocity of $4 \mathrm{~mm}$ directly under the nozzle, which is the same as the nozzle diameter, in the staggered nozzles. Table 3 shows the collision velocities used for the Re number calculations.

The relationships between $\mathrm{Re}$ and $\mathrm{Nu}$ of the slit-like nozzle and the staggered nozzle are shown in Figs. 9 and 10, respectively. These are double logarithmic plots, and Re and $\mathrm{Nu}$ of each condition are normalized as a ratio of $\mathrm{Re}$ and $\mathrm{Nu}$ under the condition of a header pressure of $5 \mathrm{kPa}$ and a nozzle distance of $10 \mathrm{~mm}$. In Fig. 9, the experimental results for the slit-like nozzle are not linear. This indicates that the heat transfer of the lateral flow along the surface is not efficient. On the other hand, in Fig. 10, the experimental results for the staggered arrangement nozzle with different gas pressures and nozzle distances lie on a single straight line. This shows that the heat transfer by the lateral flow along the steel surface is efficient. As described above, in

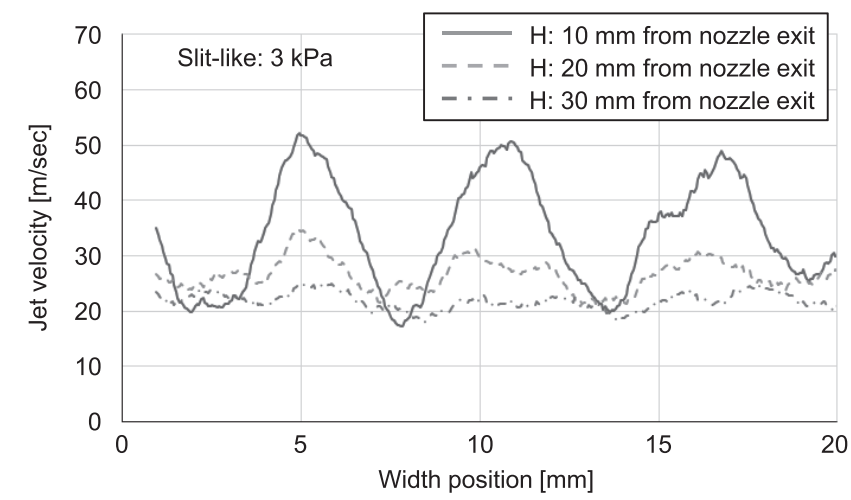

Fig. 7. Jet velocity distribution of slit-like nozzle.

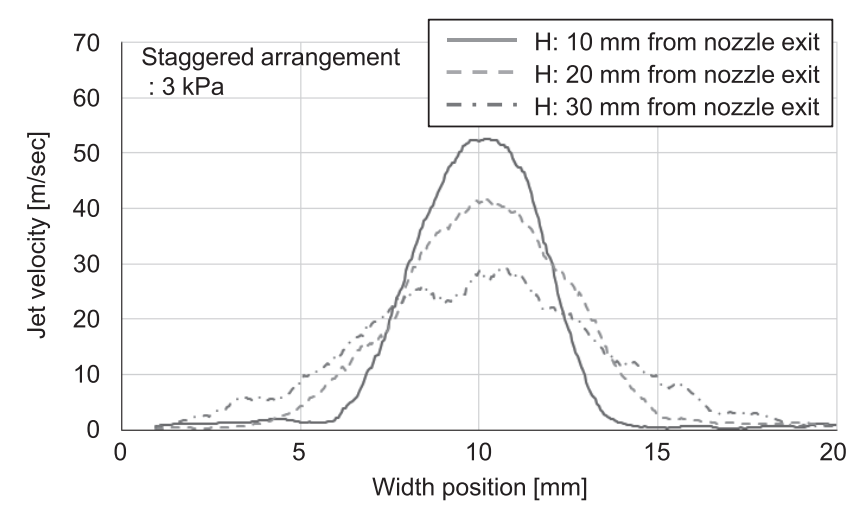

Fig. 8. Jet velocity distribution of staggered arrangement nozzle.

Table 3. Impinging jet velocities.

\begin{tabular}{ccccc}
\hline & \multicolumn{2}{c}{ Slit-like } & \multicolumn{2}{c}{ Staggered arrangement } \\
\hline $\begin{array}{c}\text { Nozzle } \\
\text { distance }\end{array}$ & $3 \mathrm{kPa}$ & $5 \mathrm{kPa}$ & $3 \mathrm{kPa}$ & $5 \mathrm{kPa}$ \\
$10 \mathrm{~mm}$ & $34.38 \mathrm{~m} / \mathrm{sec}$ & $44.44 \mathrm{~m} / \mathrm{sec}$ & $44.72 \mathrm{~m} / \mathrm{sec}$ & $57.32 \mathrm{~m} / \mathrm{sec}$ \\
$20 \mathrm{~mm}$ & $27.12 \mathrm{~m} / \mathrm{sec}$ & $34.32 \mathrm{~m} / \mathrm{sec}$ & $36.40 \mathrm{~m} / \mathrm{sec}$ & $48.29 \mathrm{~m} / \mathrm{sec}$ \\
$30 \mathrm{~mm}$ & $21.95 \mathrm{~m} / \mathrm{sec}$ & $27.22 \mathrm{~m} / \mathrm{sec}$ & $25.90 \mathrm{~m} / \mathrm{sec}$ & $37.18 \mathrm{~m} / \mathrm{sec}$ \\
\hline
\end{tabular}

the case of the slit-like nozzle, these results clarified fact that the average heat transfer coefficient decreased due to the decrease of heat transfer in the space between the nozzles.

\subsection{Numerical Analysis of Slit-like Nozzle}

In order to clarify the mechanism of the decrease in heat transfer between the nozzles, a heat transfer flow analysis was conducted. Figure 11 shows the analysis conditions. These are the same conditions as in the experiment with the slit-like nozzle. Due to the symmetry between the nozzles and at the nozzle center, symmetric boundaries can be applied to the three-dimensional model. The surrounding boundary conditions were free flow boundaries. The mesh is a polyhedral mesh, the total mesh number is about 780000 meshes under the nozzle distance condition of $30 \mathrm{~mm}$, and the minimum mesh width of the steel sheet surface layer is $100 \mu \mathrm{m}$. In this simulation, the analysis software ANSYS FLUENT v 17.1 was used. The calculation model outline is as follows.

1) Unsteady analysis of incompressible fluid

2) Consideration of turbulent flow (Realizable k- $\varepsilon$ model)

3) Use of standard wall function (EWT)

4) Consideration of gravity

The gas is air, as in the experiment. An incompressible ideal gas was applied for the air density, and the specific heat was fixed at $1006.43 \mathrm{~J} / \mathrm{kgK}$. Temperature effects were considered for thermal conductivity and the viscosity coefficient.

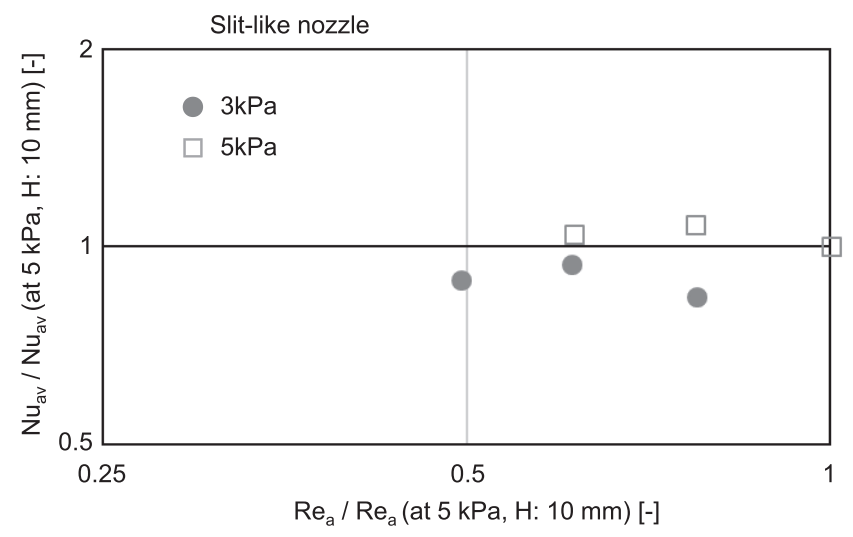

Fig. 9. Correlation of Nusselt number and Reynolds number of slit-like nozzle.

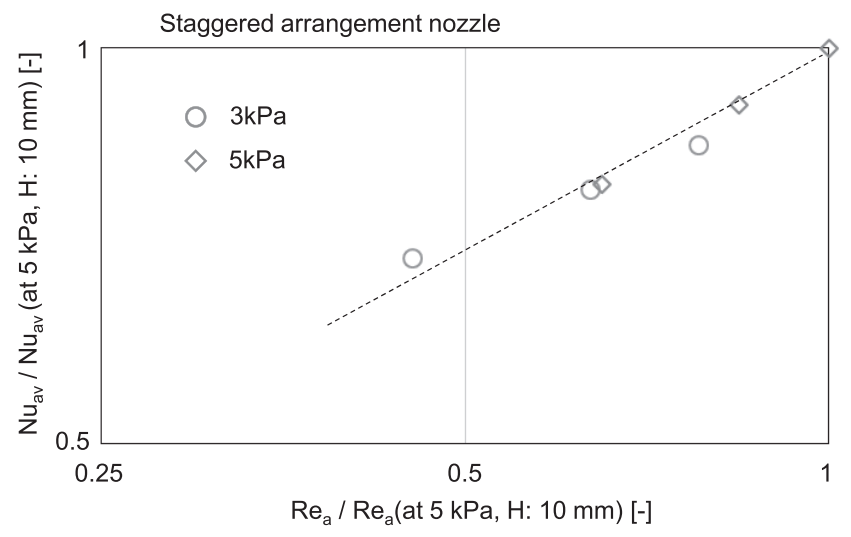

Fig. 10. Correlation of Nusselt number and Reynolds number of staggered arrangement nozzle. 

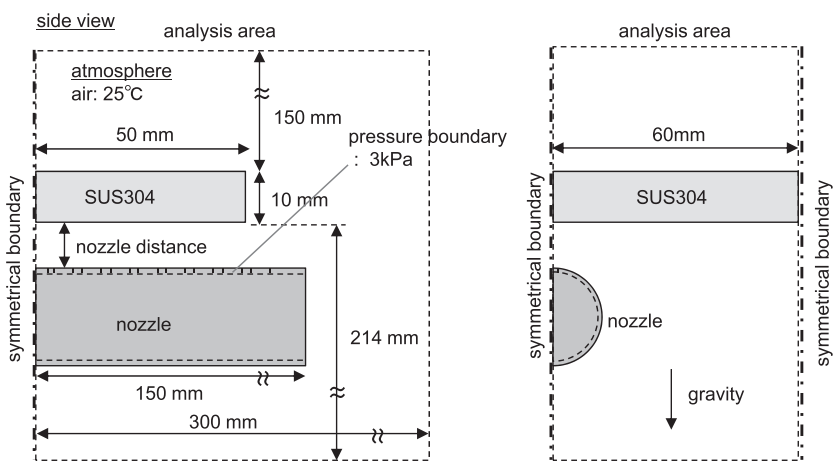

Fig. 11. Conditions used in analysis of slit-like nozzle.

Temperature effects were also considered for the specific heat and thermal conductivity of the SUS. For simplicity, the effect of radiation was not considered. A heat transfer flow analysis from the initial temperature of $500^{\circ} \mathrm{C}$ of the SUS was conducted under the above-mentioned conditions.

Figure 12 shows the SUS surface temperature history in the analysis results. For comparison, the experimental results at the nozzle distance of $10 \mathrm{~mm}$ are also shown. The experimental result is the value of the thermocouple at the depth of $2 \mathrm{~mm}$ from the surface of the SUS, and it is higher than the analysis result (surface temperature) at the stagnation point. However, between the nozzles, the cooling rate is smaller in the analysis result. As an unsteady heat transfer flow analysis was conducted, it is thought that the heat transfer accompanying the generation, development and extinction of vortices can be reproduced well. However, because the calculation region is set considering the symmetry boundaries, it is impossible to consider the heat transfer accompanying the generation of a vortex of a size which cannot be expressed at the symmetrical boundaries. Therefore, there is considered to be a possibility of underestimation of the heat transfer between the nozzles. Also, the boundary layer thickness of the flow in the stagnation region is reported to be about one hundredth of the nozzle diameter. ${ }^{5}$ Therefore, there is a possibility that the large minimum mesh width affected the results. However, for the following reasons, we think that the tendencies in the analysis can reproduce the experimental phenomena well.

The analysis results show that the cooling abilities at the nozzle distances of $10 \mathrm{~mm}$ and $20 \mathrm{~mm}$ are higher than at $30 \mathrm{~mm}$ at the stagnation point, but there is almost no difference between $10 \mathrm{~mm}$ and $20 \mathrm{~mm}$. The reason is thought to be that the effect of velocity attenuation is slight in the region of the potential core. Generally, in a two-dimensional jet flow, the length of the potential core is about $5 \mathrm{D}$ to 6 $\mathrm{D}^{11,12)}$ of the nozzle diameter $\mathrm{D}$. Thus, at a nozzle distance of $30 \mathrm{~mm}$, the cooling ability decreases due to the effect of velocity attenuation. These tendencies are in good agreement with the experimental results in Fig. 5.

In addition, it is understood the cooling abilities at the $1 / 4$ point and $1 / 2$ point between the nozzles improve as the nozzle distance becomes longer. Since these tendencies are in good agreement with the experimental results, we think that analysis results can reproduce the real phenomena with high accuracy.

In order to consider the flow analysis results, the characteristics of the impinging jet ${ }^{5,6)}$ will be explained by using
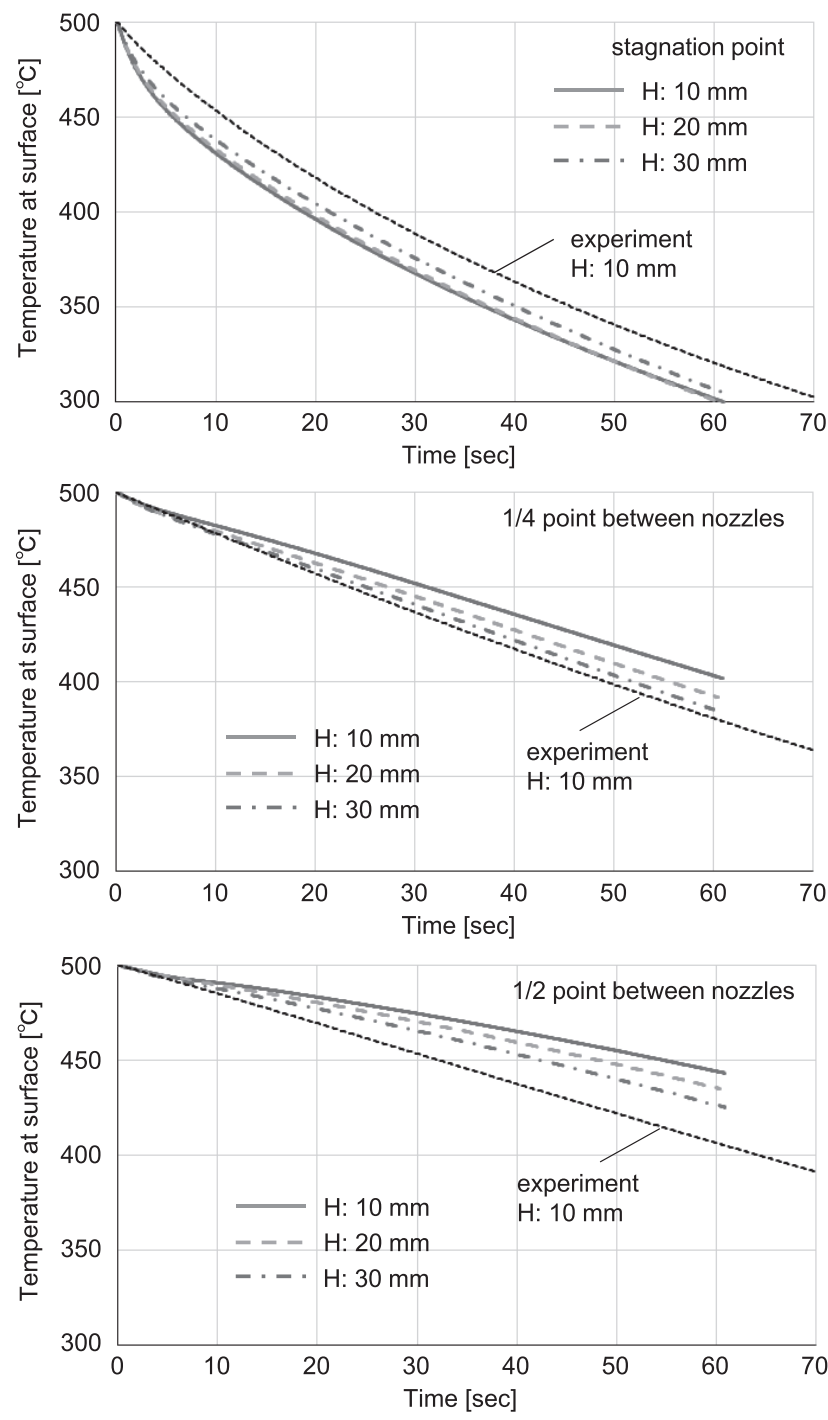

Fig. 12. Temperature history in analysis (slit-like nozzle: $3 \mathrm{kPa}$ ).

the schematic diagram in Fig. 13. The uniform velocity jet from the nozzle spreads in the atmosphere and gradually forms a mild velocity distribution according to the mass and energy conservation law. This region is called the flow development region, and in this region, there is a wedge-shaped potential core where the average velocity does not decrease at all. When the jet reaches the wall surface, the velocity component in the nozzle axial direction rapidly decreases and changes to a lateral flow along the wall surface (wall jet).

In order to verify the cause of the decrease of cooling ability between the nozzles under the condition of nozzle proximity, the average flow velocity in the width direction on the SUS surface is shown in Fig. 14. The horizontal axis is the position in the direction of the long nozzle span, and $0 \mathrm{~mm}$ is the stagnation point. At the stagnation point, the wall jet velocity increases as the nozzle distance decreases. However, in the region over $10 \mathrm{~mm}$ from the stagnation point between the nozzles, the average flow velocity becomes larger as the distance between the nozzle and the SUS surface increases, and there is almost no difference between the average velocities at the $1 / 2$ point between the nozzles (60 $\mathrm{mm}$ position). Between the nozzles, the heat transfer by the lateral flow decreases because the decrease of 


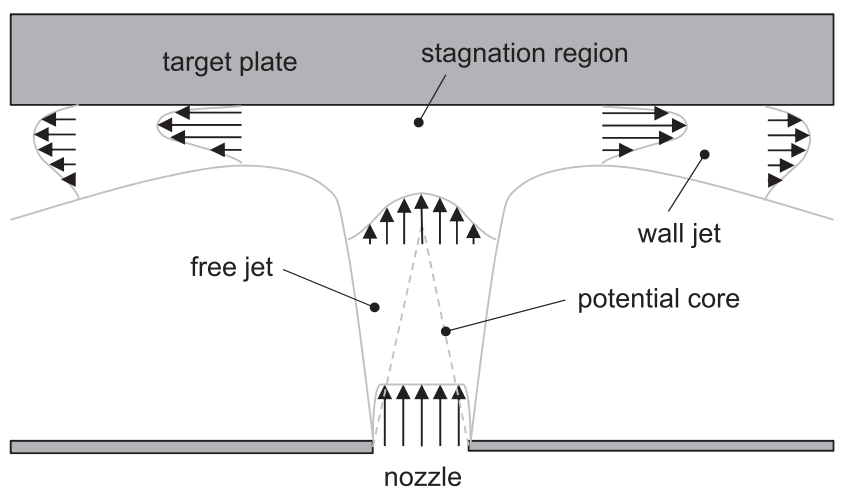

Fig. 13. Schematic illustration of flow characteristics of impinging jet.

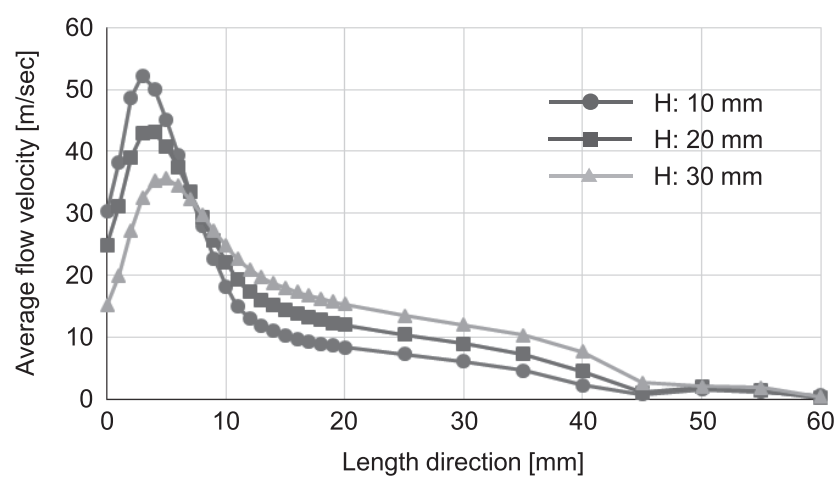

Fig. 14. Flow velocity distribution on cooled surface (slit-like nozzle).

the lateral flow velocity is large, and as a result, the cooling ability decreases. Under the condition of nozzle proximity, although the impinging jet velocity is large, the lateral flow velocity decreases drastically. As the reason for this, it is thought that the interference of the jets in the short span direction of the nozzles affects the lateral flow velocity, causing this large decrease.

The jet interference in the short span direction of the nozzles will be explained by using Fig. 15. In the multi-hole nozzle, when the distance between the nozzle and the cooled surface is long, the jets from the single nozzles combine and become uniform, as shown on the left in Fig. 15. However, when the nozzle distance is short, the jets impinge on the surface without diffusing greatly, while maintaining a potential core, as shown at the right in Fig. 15. Under this condition, lateral flow impingement occurs between the short spans of the nozzles, the lateral flow component is canceled, and the jet velocity potential along the surface in the long direction of the nozzle span decreases. From the jet velocity distribution result in Fig. 7, it is also understood that the jet does not become uniform, but rather keeps a velocity distribution, at $10 \mathrm{~mm}$ from the nozzle tip.

In an experiment under the condition of $H / D=3$ with an air jet at $130^{\circ} \mathrm{C}$, Grenson et al. ${ }^{13)}$ reported that the velocity component of the lateral flow is accelerated in the range of $r / D$ from about 1.5 to 1.7 , where $r$ is the distance from the stagnation point. In this research, $r / D$ is 0.875 for the short span of the nozzles. Jet interference occurs before the wall jet is completely developed, that is, before the velocity in the nozzle axial direction shifts completely to the velocity

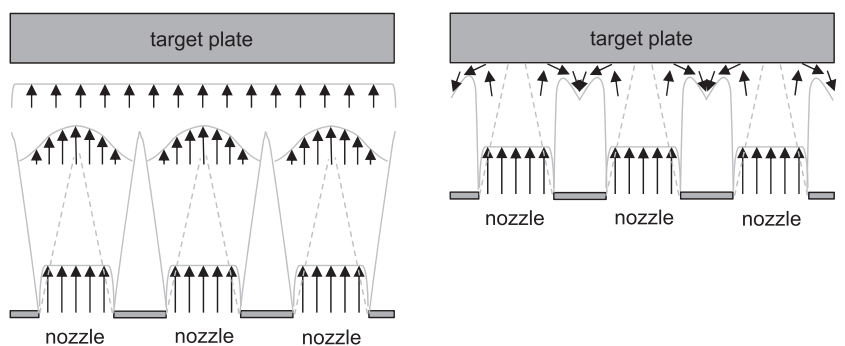

Fig. 15. Flow characteristics by distance between nozzle and cooled surface.

in the direction along to the surface, and it is thought that this has the effect of reducing the jet velocity potential along the surface.

From the above consideration, the mechanism of the decrease in cooling ability under the condition of nozzle proximity with a multi-hole slit-like nozzle was clarified.

\subsection{Numerical Analysis of Staggered Arrangement Nozzle}

In the case of the staggered arrangement nozzle, a heat transfer flow analysis was conducted in order to verify whether a decrease in the cooling ability between the nozzles occurred. The analysis conditions are shown in Fig. 16. The conditions are the same as the experimental conditions of the staggered arrangement nozzle. The mesh is a polyhedral mesh, the total mesh number is about 1.36 million meshes for the nozzle distance condition of $30 \mathrm{~mm}$, and the minimum mesh width of the steel sheet surface layer is $100 \mu \mathrm{m}$. The other conditions are the same as those in the slit-like nozzle analysis.

The analysis results of the temperature history of the SUS surface are shown in Fig. 17. For comparison, the experimental results at the nozzle distance of $10 \mathrm{~mm}$ are also shown. As in the case of the slit-like nozzle, the cooling rates between the nozzles were smaller in the analysis results than in the experiments. For this reason, the possibility of underestimation of heat transfer accompanying the vortex which cannot be expressed in the symmetrical boundary condition, and the influence of the mesh width are conceivable. However, for the following reasons, it is thought that the analysis can reproduce the experimental phenomena well.

In the analysis results, temperature differences (temperature distribution) are generated from the start of cooling at the stagnation point, $1 / 4$ point and $1 / 2$ point of the nozzles due to the difference of cooling ability, but at all points, cooling efficiency increased as the nozzle distance $\mathrm{H}$ decreased. This is in good agreement with the experimental results in Fig. 6.

In order to consider the influence of the jet velocity on the surface of the SUS between the nozzles, the average jet velocity at the same distance from the nozzle center (average velocity on a concentric circle) is shown in Fig. 18. The jet velocity decreases at the stagnation point $(0 \mathrm{~mm}$ position), but the maximum velocity near the stagnation point increase as the nozzle distance becomes closer. In addition, it can be understood that the jet velocity between the nozzles also increases as the nozzle distance decreases.

With the staggered arrangement, the jet velocity between 


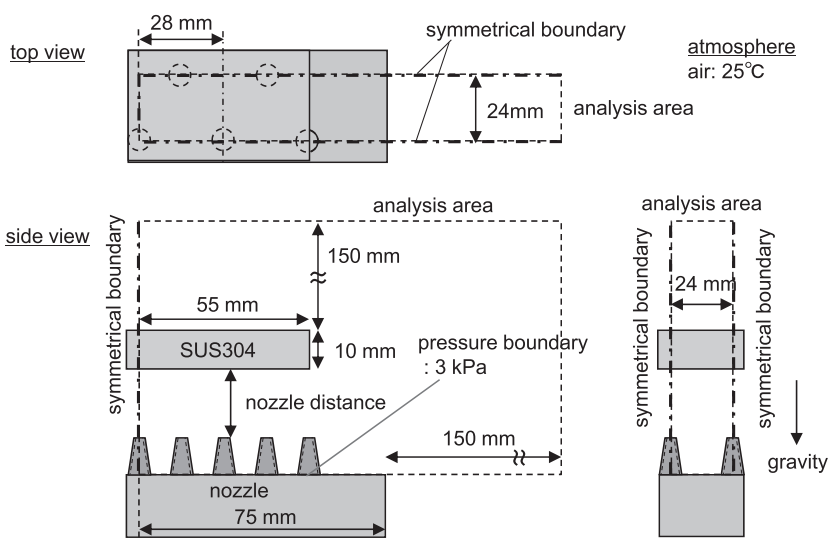

Fig. 16. Conditions used in analysis of staggered arrangement nozzle.
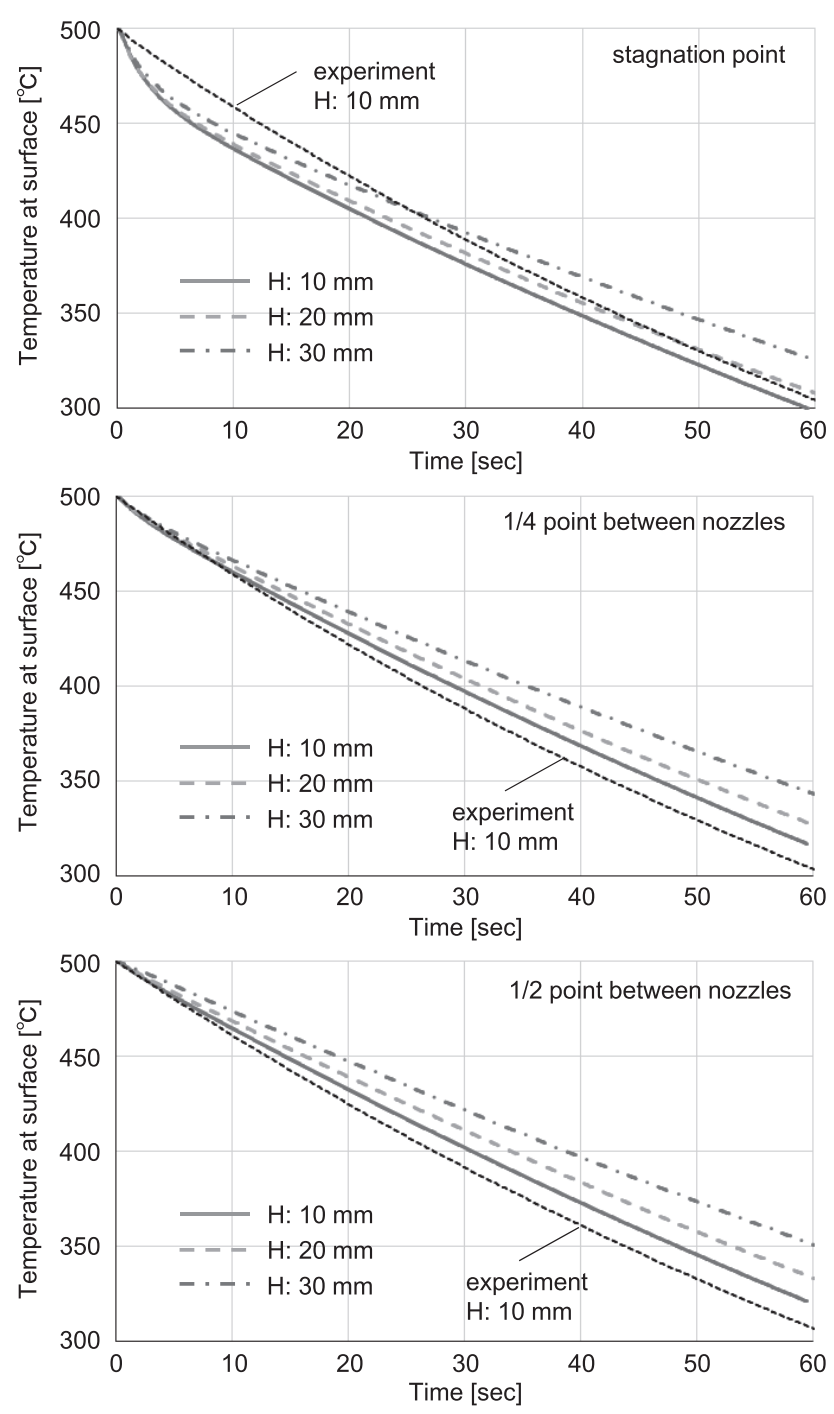

Fig. 17. Temperature history in analysis (staggered arrangement nozzle: $3 \mathrm{kPa}$ ).

the nozzles also increases; this is consistent with the experimental result that the cooling ability improves as the distance from the nozzle to the surface decreases, On the other hand, the increment of the jet velocity on the surface due to nozzle proximity is slightly lower when the distance is decreased from $20 \mathrm{~mm}$ to $10 \mathrm{~mm}$ compared to $30 \mathrm{~mm}$

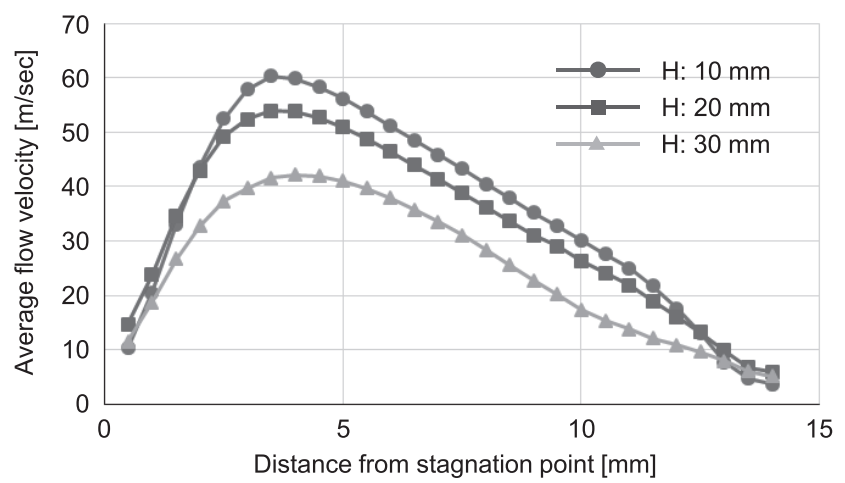

Fig. 18. Flow velocity distribution on cooled surface (staggered arrangement nozzle).

to $20 \mathrm{~mm}$. At the nozzle distance of $30 \mathrm{~mm}$, the impinging jet velocity decreases as a result of velocity attenuation. However, it is thought that velocity attenuation is small at $20 \mathrm{~mm}$, and as a result, the impinging jet velocity is much the same as that at the nozzle distance of $10 \mathrm{~mm}$.

The nozzle distance of $20 \mathrm{~mm}$ is 5 times the nozzle diameter of $4 \mathrm{~mm}$, and thus is in the region where the potential core exists. Therefore, when the distance from the nozzle to the surface is reduced from $20 \mathrm{~mm}$ to $10 \mathrm{~mm}$, it is considered that velocity attenuation is small, and improvement of the cooling ability is also small. These results suggest that it is necessary to consider the potential core region in the Martin prediction formula under the condition of nozzle proximity of the staggered nozzle.

In the Martin equation, the application range of the multi-hole nozzle is set to $2 \leqq \mathrm{H} / \mathrm{D}$ (nozzle distance/nozzle diameter) $\leqq 12$. These experimental results show that deviation tends to increase in the region of $H / D \leqq 5$. $H / D \fallingdotseq 5$ to 6 is the region where the potential core disappears, and the transition from the flow development region of the jet to the fully-developed flow region occurs. It is known that the characteristics of a two-dimensional impinging jet, including the lateral flow, change rapidly from around $\mathrm{H} / \mathrm{D} \fallingdotseq 5 .{ }^{12}$ ) Therefore, it is thought that the cooling ability also changes around $\mathrm{H} / \mathrm{D} \fallingdotseq 5$. In the region of $\mathrm{H} / \mathrm{D} \leqq 5$ in the Martin equation, it can be said that it is a necessary to correct the equation for $5<\mathrm{H} / \mathrm{D} \leqq 12$. It is concluded that the main cause of the difference between the Martin prediction formula and the experiments in the case of nozzle proximity is a change in the characteristics of the impinging jet.

\section{Final Remarks}

In order to predict the cooling ability of a gas jet simply and accurately, a gas jet cooling experiment and nozzle flow analyses were conducted with various nozzle arrangements, distances, heights and shapes. The effect of each factor was investigated and compared with the results of calculations by Martin's dimensionless characteristic equation, ${ }^{3)}$ which is known as a simple prediction formula for the heat transfer coefficient of a gas jet, and the following knowledge was obtained.

(1) In the slit-like nozzle, as the nozzle approaches the region where the potential core is present, which is the development region of the jet, the heat transfer efficiency between the nozzles decreases notably, and as a result, the 
average heat transfer coefficient decreases.

(2) In predicting the cooling ability of a slit-like nozzle, prediction accuracy is improved if the difference between the contraction flow ratio of the slit nozzle and the multi-hole nozzle is corrected and reflected in the Martin prediction equation (prediction formula for slit nozzle).

(3) From the heat transfer flow analysis of the slit-like nozzle, it was understood that the gas flow velocity on the steel surface between the nozzles decreased due to nozzle proximity. It is thought that this velocity decrease is caused by the decrease of the velocity potential of the lateral flow on the surface, because the jets from each of the nozzles arranged at short spans impinge on the steel surface and interfere before a uniform jet can form sufficiently.

(4) In a staggered arrangement nozzle, the heat transfer coefficient increases with nozzle proximity, but in the region of (nozzle distance)/(nozzle diameter) $\leqq 5$, the deviation between the experiment and the Martin prediction formula increases. That is, the Martin prediction formula tends to overestimate the increase of the heat transfer coefficient due to nozzle proximity.

(5) The Martin prediction formula is able to predict the value and the change of the heat transfer coefficient of a gas jet with reasonable accuracy. Moreover, a further improvement in prediction accuracy can be achieved by taking into account the decrease of the flow velocity due to the contraction flow and pressure loss, velocity attenuation, and correction to the potential core region in cooling under a condition of nozzle proximity.

\section{REFERENCES}

1) K. S. Choo and S. J. Kim: Int. J. Heat Mass Transf., 53 (2010), 320.

2) S. V. Prabhu, P. Gulati and V. Katti: Int. J. Therm. Sci., 48 (2009), 602.

3) H. Martin: Adv. Heat Transf., 13 (1977), 1.

4) S. Oomori: Mitsubishi Heavy Ind. Tech. Rev., 9 (1972), 380.

5) B. Weigand and S. Spring: Heat Transf. Res., 42 (2011), 101.

6) N. Zuckerman and N. Lior: Adv. Heat Transf., 39 (2006), 565.

7) M. Attalla and E. Specht: Heat Mass Transf., 45 (2009), 537.

8) V. Narayanan, J. Seyed-Yagoobi and R. H. Page: Int. J. Heat Mass Transf., 47 (2004), 1827.

9) The Iron and Steel Institute of Japan: Kouzai no kyousei reikyaku, ISIJ, Tokyo, (1978).

10) L. F. G. Geers, M. J. Tummers, T. J. Bueninck and K. Hanjalic: Int. J. Heat Mass Transf., 51 (2008), 5389.

11) N. Rajaratnam: Turbulent Jets, Elsevier Scientific Publishiing, Amsterdam, (1976).

12) S.Iida: J. Jpn. Coat. Technol. Assoc., 50 (2015), 388.

13) P. Grenson, O. Leon, P. Reulet and B. Aupoix: Int. J. Heat Mass Transf., 102 (2016), 801 